\title{
Non-Invasive Measurement of Stress Levels in Knee Implants Using a Magnetic-Based Detection Method
}

\author{
David Okhiria, Turgut Meydan, and Paul I. Williams \\ Wolfson Centre for Magnetics, School of Engineering, Cardiff University, Cardiff CF24 3AA, U.K.
}

\begin{abstract}
A knee replacement surgery (arthroplasty) has become prevalent worldwide and has a high success rate over the short to medium term. In some cases, especially over the longer term, implant degradation can develop due to the deterioration of the ultra-high molecular weight polyethylene (UHMWPE) tibial insert. Unfortunately, there are no satisfactory techniques currently available for assessing implant integrity and predicting failure. This paper describes a possible solution to this problem by using a non-invasive, electromagnetic method for monitoring implant integrity. This approach utilizes the magnetoelastic property of amorphous ribbon, which when stressed causes an inductance change in a nearby magnetizing winding. Amorphous ribbons encased in UHMWPE disks, to simulate a knee insert, were subjected to varying tensile stresses under an applied ac magnetic field. A correlation between total circuit impedance and applied stress was observed. The results obtained demonstrate that the proposed sensor has sufficient sensitivity for measuring typical stress levels associated with the axial forces in tibial inserts.
\end{abstract}

Index Terms-Amorphous materials, biomedical applications, knee implants, magnetic sensor.

\section{INTRODUCTION}

$\mathbf{T}$ ECHNOLOGICAL advancements in knee arthroplasty have made it prevalent around the world, with over half a million primary procedures performed in England and Wales alone during the last decade [1]. It is a complex surgical procedure, which requires the proper alignment of the new and existing articular surfaces, in combination with the accurate repositioning of the associated tissues. The number of total knee replacement (TKR) surgeries is constantly rising due to increased population and life expectancy rates, as well as a general decline in the age at the time of initial TKR. As a result of this, extensive research is being carried out on how to improve the survival rates and prolong the lifespan of the implant [2]. In addition, early detection of high wear rates allows for anticipatory treatment, which could eventually prevent premature implant failure.

The most probable cause of premature implant failure is aseptic loosening, which is a severe physiological response to foreign debris in the joint. This debris is generated from abrasive wear in the ultra-high molecular weight polyethylene (UHMWPE) of the tibial insert, due to malpositioning of the articular surfaces and the high forces acting in the joint [3], [4]. Therefore, effective monitoring of the active stresses within the UHMWPE insert can provide a clearer perception of knee biomechanics, and provide real-time observation on the condition of the knee implant.

Although previous attempts have been made at designing implantable monitoring systems [5]-[8], many are only usable during the surgical process or require modifications to the existing implant architecture [9]. In this paper, amorphous magnetostrictive ribbons were integrated into UHMWPE as

Manuscript received September 7, 2015; revised January 22, 2016; accepted January 22, 2016. Date of publication January 27, 2016; date of current version April 15, 2016. Corresponding author: T. Meydan (e-mail: meydan@cardiff.ac.uk).

Color versions of one or more of the figures in this paper are available online at http://ieeexplore.iee.org.

Digital Object Identifier 10.1109/TMAG.2016.2521421 sensors for measuring the compressive loads acting upon the insert. The fundamental design and principle of operation are presented alongside the results obtained from initial tests carried out.

\section{THEORY}

Amorphous ferromagnetic materials are an important class of materials, which have been exploited in a diverse range of magnetic applications [10]-[14]. They are usually manufactured as a ribbon or wire by melt-spinning and rapid quenching techniques to form non-crystalline alloys of iron, cobalt or nickel, and metalloids (mostly boron and silicon). Ferromagnetic materials are composed of singular irregularly magnetized domains, which are oriented, such that the net magnetization within the material is approximately zero. Any external stress on the material causes a realignment of these domains, and the associated strain introduces magnetoelastic anisotropy in the sample, leading to a change in permeability.

The variation in flux density and magnetic permeability can be observed using an induction coil placed near or around the magnetic material. Assuming a uniform flux density distribution in the region enclosed by the coils, and from the inductance approximation, it follows that the inductance of the coil proportionally varies with the change in permeability. The presence of the ferromagnetic material increases the flux density for a specified current value, and this alters the inductance of the coil. These variations in inductance can be measured using a parallel tuned circuit with known capacitance, where the magnitude of the resultant impedance is maximal at the resonant frequency.

\section{MethoD}

As-cast highly magnetostrictive amorphous ribbons (Metglas 2605SC) were utilized due to their high magnetomechanical coupling coefficient and stress sensitivity. The $25 \mu \mathrm{m}$ thick ribbons were cut into rectangular strips measuring $48 \mathrm{~mm} \times 7 \mathrm{~mm}$, and a total of four strips were stacked and glued together with an epoxy resin to obtain a solid core (with a demagnetizing factor of $\sim 0.006$ ). 


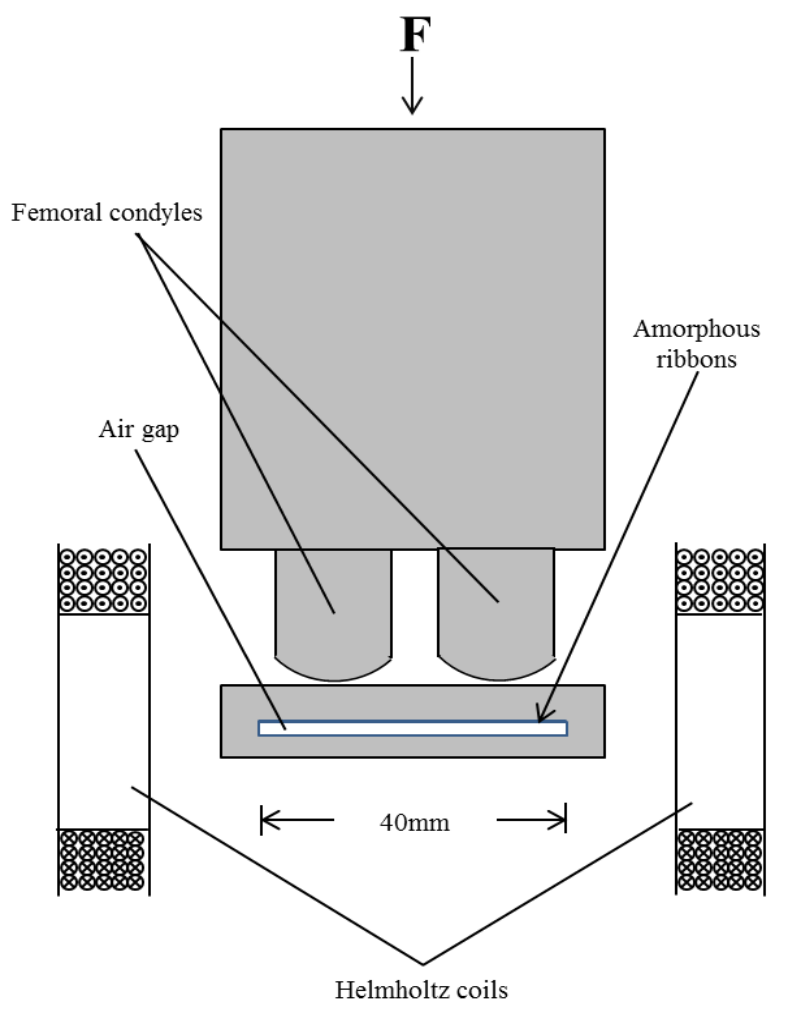

(a)

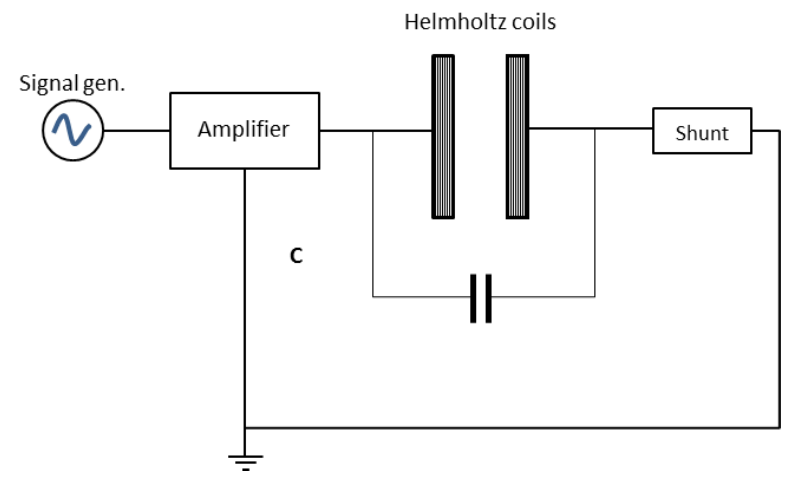

(b)

Fig. 1. (a) Cross-sectional view showing the position of polypropylene condyles and UHMWPE insert within the Helmholtz coils. (b) Schematic of the electrical measurement circuit.

The resulting amorphous sample was sandwiched between two $5 \mathrm{~mm}$ thick circular UHMWPE disks measuring $50 \mathrm{~mm}$ in diameter. To allow for the deflection of the ribbons when stressed, a $2 \mathrm{~mm}$ deep rectangular trench measuring $40 \mathrm{~mm} \times 8 \mathrm{~mm}$ was centrally bored on the inner surface of the bottom disk. The low coefficient of friction of UHMWPE makes bonding difficult. This problem was circumvented by treating the surfaces with a polyolefin primer prior to bonding with a cyanoacrylate-based adhesive. A profile of the resulting insert design is shown in Fig. 1(a), where it can be noted that only a $40 \mathrm{~mm}$ section of ribbon actually experiences deflection due to strain. To simulate the femoral condyles, a model was fabricated from polypropylene (which has a higher compressive strength than UHMWPE) with the spherical

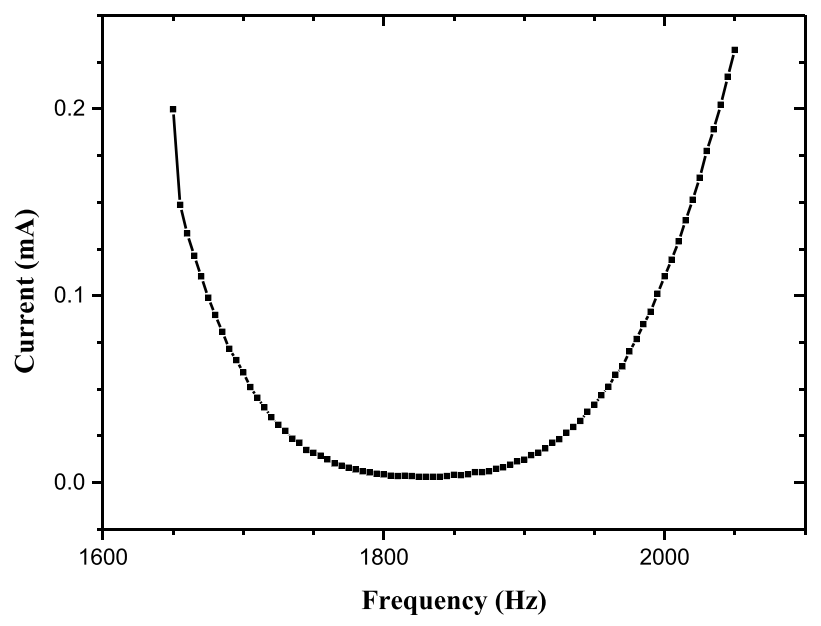

Fig. 2. Frequency sweep of the tank circuit showing resonant frequency.

articulating surfaces of radius $20 \mathrm{~mm}$. This radius was selected in order to achieve initial contact stresses, which fall within the reported range after TKR (about 30-60 MPa) [15], [16]. The inserts were subjected to axial compressive loads varying from 100 to $5000 \mathrm{~N}$, using a Zwick Roell Z050 materials testing machine, in the presence of an AC magnetic field generated by a pair of Helmholtz coils measuring $22 \mathrm{~cm}$ in diameter and with 1470 turns each. The coils were fed by a Krohn-Hite model 7500 wideband power amplifier and were connected in parallel with a $0.01 \mu \mathrm{F}$ capacitor to form a tuned $(L C)$ circuit. The resonant frequency, the voltage across the tuned circuit, and the line current through the circuit for each value of applied load were measured and recorded. The voltage and current measurements were automated using two Agilent 34401A digital multimeters controlled using LabVIEW software. A diagram of the electrical circuit is shown in Fig. 1(b).

\section{RESUlts AND Discussion}

A frequency sweep of the tuned circuit indicated that it resonated at $1.82 \mathrm{kHz}$, as shown in Fig. 2. It was noted that the voltage across the tuned circuit remained constant over the frequency range. Since the associated strains are small and both articulating surfaces are non-conforming, the value of applied stress was approximated using the Hertzian theory of non-adhesive contact between a sphere and an elastic half-space [17]. The radius of the contact area between each condyle of and the surface of the insert is given by

$$
a=\sqrt[3]{\frac{3 F R}{4 E^{*}}}
$$

where $F$ is the applied force per condyle in Newtons. In this case, the effective radius $(R)$ is the radius of the condyles, since the insert is flat. The reduced modulus, $E^{*}$, can be defined as

$$
E^{*}=\left(\frac{1-v_{1}^{2}}{E_{1}}+\frac{1-v_{2}^{2}}{E_{2}}\right)^{-1}
$$

where $E_{1,2}$ and $v_{1,2}$ are the elastic moduli and Poisson's ratios for the condyles and the insert, respectively. The maximum 
TABLE I

Calculated Contact Pressure for Various VALUES OF APPLIED ForCE

\begin{tabular}{ccc}
\hline $\begin{array}{c}\text { Total applied } \\
\text { force (Newtons) }\end{array}$ & $\begin{array}{c}\text { Radius of contact } \\
\text { area circle per } \\
\text { condyle (mm) }\end{array}$ & $\begin{array}{c}\text { Max. contact } \\
\text { pressure per } \\
\text { condyle (MPa) }\end{array}$ \\
\hline 100 & 1.08 & 20.54 \\
200 & 1.36 & 25.88 \\
300 & 1.55 & 29.62 \\
400 & 1.71 & 32.60 \\
500 & 1.84 & 35.12 \\
1000 & 2.32 & 44.25 \\
2000 & 2.93 & 55.75 \\
3000 & 3.35 & 63.82 \\
4000 & 3.69 & 70.24 \\
5000 & 3.97 & 75.66 \\
\hline
\end{tabular}

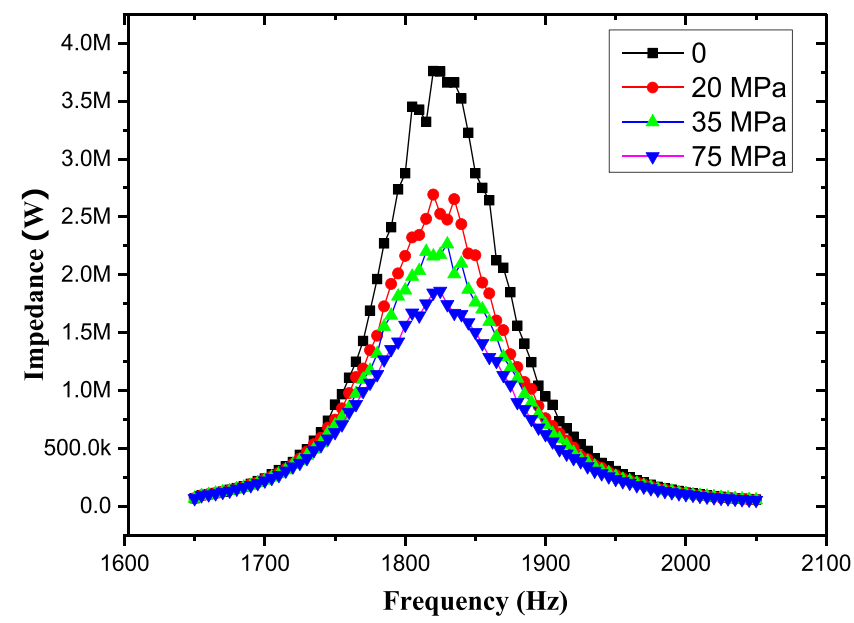

Fig. 3. Measured impedance spectrum for various values of contact pressure.

contact pressure on the insert is given by

$$
p_{0}=\frac{3 F}{2 \pi a^{2}} \text {. }
$$

The forces were centrally applied to the fabricated condyles, and hence, the force on each condyle was assumed to be equal to half of the total applied. The elastic moduli of polypropylene and UHMWPE were taken as 1.5 and $0.7 \mathrm{GPa}$, respectively, while Poisson's ratio for both materials was set as 0.45 . The corresponding contact pressure values at the surface of the insert for the applied forces are presented in Table I. From the measured voltage and current values, the magnitude of the $L C$ circuit impedances at different frequencies was calculated using Ohm's law and plotted, as shown in Fig. 3. As the insert wears out due to abrasion, the load bearing surface gets thinner; hence, the deflection in the ribbons due to similar axial loading is more. This introduces more strain and ultimately, a proportional decrease in initial permeability.

It was observed that increasing the contact pressure while keeping the frequency constant resulted in a steady decay in the measured $L C$ circuit impedance. For better comparison, the normalized values of the measured impedance and the corresponding contact pressure at different frequencies are plotted, as shown in Fig. 4. The variation remained linear

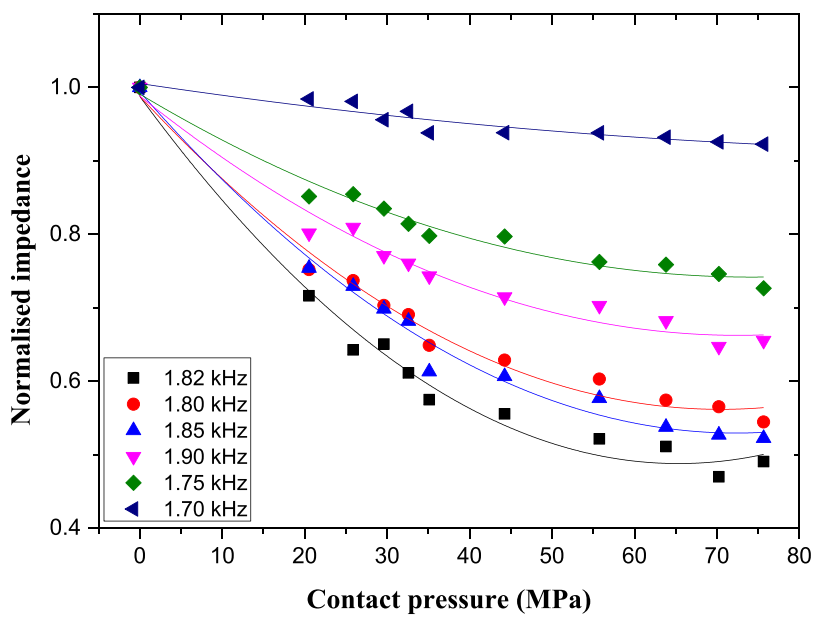

Fig. 4. Normalized impedance versus contact pressure at various frequencies $\left(R^{2}>0.9\right)$.

for contact pressure up to about $40 \mathrm{MPa}$ before tapering off, and the sensitivity was highest at resonance (evidenced by the largest decay rate of $-0.046 \Omega / \mathrm{Pa}$ ) but this sharply declined as the frequency deviated from this value. Although the magnitude of the impedance considerably varied with applied pressure (over the range of about $2 \mathrm{MHz}$ ), the changes in the actual inductance were quite small (a $2 \mathrm{mH}$ variation). A consequence of this was that the resonant frequency remained fairly constant over the range measured. This can be overcome by using coils with lower inductance, such that small inductance changes will produce a significant shift in the resonant frequency. In addition, using more sensitive meters with higher resolution will enable easier detection of resonance changes down to a few Hertz. Greater sensitivity can be realized by increasing the dimensions of the amorphous ribbons utilized and eliminating random intrinsic stresses to obtain a uniaxial domain structure.

The repeatability has been reported in terms of the standard deviation of the measured quantities at each frequency. The maximum deviation was $\sim 0.08$ and this occurred when the insert was initially stressed. As the contact pressure increased to about $29 \mathrm{MPa}$, the deviation dropped to less than 0.04 , and increasing the contact pressure beyond that dropped the deviation even further. Similarly, the maximum value of the normalized error of the impedance at the point of initial stress was 0.1 (at the resonant frequency) but this steadily dropped with increased loading to 0.06 . This reported uncertainty is based on a standard uncertainty multiplied by a coverage factor $k=1$, which provides a confidence level of $\sim 68 \%$.

Additional research can be carried out to determine the optimal positioning of the amorphous ribbons within the insert for maximum stress transfer. The data obtained establish that the setup is suitable for measuring the contact pressure due to the combined axial forces, which the insert is subjected to, up to about $70 \mathrm{MPa}$, which falls within the reported stresses after TKR.

\section{CONCLUSION}

This paper described a magnetic-based detection method in which amorphous ribbons were used to determine the stress 
levels in knee implants. Wear can be related to the increased loading on the UHMWPE tibial insert, and this resulted in a proportionate drop in the magnetizing winding inductance as the ribbon permeability changed. The method employed is passive, non-invasive, and requires minimal modification to existing insert designs. More importantly, this approach eliminates the need for secondary windings; hence, it can be used for in vivo observation even without additional circuitry. Effective and timely monitoring of implant stress can prolong their longevity, thereby reducing the associated replacement costs.

\section{ACKNOWLEDGMENT}

The authors would like to thank A. Rankmore, the Technical Supervisor, from the School Mechanical Workshop for the assistance with the fabrication of the polypropylene model. They would also like to thank H. Lane, the Technical Supervisor, and I. King from the School Civil Engineering Workshop for their guidance on the use of the stress testing machine.

\section{REFERENCES}

[1] National Joint Registry for England, Wales and Northern Ireland. (2014). 11th Annual Report. [Online]. Available: http://www.njrreports.org.uk/

[2] B. Heinlein et al., "ESB clinical biomechanics award 2008: Complete data of total knee replacement loading for level walking and stair climbing measured in vivo with a follow-up of 6-10 months," Clin. Biomech., vol. 24, no. 4, pp. 315-326, May 2009.

[3] D. D. D’Lima, S. Patil, N. Steklov, J. E. Slamin, and C. W. Colwell, Jr., "Tibial forces measured in vivo after total knee arthroplasty," J. Arthroplasty, vol. 21, no. 2, pp. 255-262, Feb. 2006.

[4] A. Arami et al., "Instrumented prosthesis for knee implants monitoring," in Proc. IEEE Int. Conf. Autom. Sci. Eng., Aug. 2011, pp. 828-835.
[5] S. J. G. Taylor, P. S. Walker, J. S. Perry, S. R. Cannon, and R. Woledge, "The forces in the distal femur and the knee during walking and other activities measured by telemetry," J. Arthroplasty, vol. 13, no. 4, pp. 428-437, Jun. 1998.

[6] B. Kirking, J. Krevolin, C. Townsend, C. W. Colwell, Jr., and D. D. D'Lima, "A multiaxial force-sensing implantable tibial prosthesis," J. Biomech., vol. 39, no. 9, pp. 1744-1751, 2006.

[7] B. Heinlein, F. Graichen, A. Bender, A. Rohlmann, and G. Bergmann, "Design, calibration and pre-clinical testing of an instrumented tibial tray," J. Biomech., vol. 40, no. 1, pp. S4-S10, 2007.

[8] L. Mohanty, S. C. Tjin, D. T. T. Lie, S. E. C. Panganiban, and P. K. H. Chow, "Fiber grating sensor for pressure mapping during total knee arthroplasty," Sens. Actuators A, Phys., vol. 135, no. 2, pp. 323-328, Apr. 2007.

[9] W. Hasenkamp et al., "Design and test of a MEMS strain-sensing device for monitoring artificial knee implants," Biomed. Microdevices, vol. 15, no. 5, pp. 831-839, Oct. 2013.

[10] R. Hasegawa, "Applications of amorphous magnetic alloys in electronic devices," J. Non-Crystalline Solids, vol. 287, nos. 1-3, pp. 405-412, Jul. 2001.

[11] P. Marín and A. Hernando, "Applications of amorphous and nanocrystalline magnetic materials," J. Magn. Magn. Mater., vols. 215-216, pp. 729-734, Jun. 2000.

[12] K. J. Overshott and T. Meydan, "Unmagnetized amorphous ribbon transducers," IEEE Trans. Magn., vol. 20, no. 5, pp. 948-950, Sep. 1984.

[13] K. Harada, I. Sasada, T. Kawajiri, and M. Inoue, "A new torque transducer using stress sensitive amorphous ribbons," IEEE Trans. Magn., vol. 18, no. 6, pp. 1767-1769, Nov. 1982.

[14] J. Seekircher and B. Hoffmann, "New magnetoelastic force sensor using amorphous alloys," Sens. Actuators A, Phys., vol. 22, nos. 1-3, pp. 401-405, Jun. 1990.

[15] J. A. Szivek, P. L. Anderson, and J. B. Benjamin, "Average and peak contact stress distribution evaluation of total knee arthroplasties," J. Arthroplasty, vol. 11, no. 8, pp. 952-963, Dec. 1996.

[16] M. S. Kuster, G. A. Wood, G. W. Stachowiak, and A. Gächter, "Joint load considerations in total knee replacement," Bone Joint J., vol. 79, no. 1, pp. 109-113, 1997.

[17] K. L. Johnson, Contact Mechanics. Cambridge, U.K.: Cambridge Univ. Press, 1985, pp. 90-93. 